\title{
Rigorous Determination of the Stoichiometry of Protein Phosphorylation Using Mass Spectrometry
}

\author{
Hannah Johnson, ${ }^{a}$ Claire E. Eyers, ${ }^{\text {a }}$ Patrick A. Eyers, ${ }^{b}$ Robert J. Beynon, ${ }^{c}$ \\ and Simon J. Gaskell ${ }^{\mathrm{a}}$ \\ ${ }^{a}$ Michael Barber Centre for Mass Spectrometry, Manchester Interdisciplinary Biocentre, School of Chemistry, \\ University of Manchester, Manchester, United Kingdom \\ b Yorkshire Cancer Research Institute for Cancer Studies, University of Sheffield, Sheffield, United Kingdom \\ c Proteomics and Functional Genomics Group, Faculty of Veterinary Science, University of Liverpool, \\ Liverpool, United Kingdom
}

Quantification of the stoichiometry of phosphorylation is usually achieved using a mixture of phosphatase treatment and differential isotopic labeling. Here, we introduce a new approach to the concomitant determination of absolute protein concentration and the stoichiometry of phosphorylation at predefined sites. The method exploits QconCAT to quantify levels of phosphorylated and nonphosphorylated peptide sequences in a phosphoprotein. The nonphosphorylated sequence is used to determine the absolute protein quantity and serves as a reference to calculate the extent of phosphorylation at the second peptide. Thus, the stoichiometry of phosphorylation and the absolute protein concentration can be determined accurately in a single experiment. (J Am Soc Mass Spectrom 2009, 20, 2211-2220) (C) 2009 American Society for Mass Spectrometry

$\mathrm{R}$ eversible protein phosphorylation is one of the most important post-translational modifications, regulating signaling in both prokaryotic and eukaryotic cells. The incorporation of a phosphate group at specific amino acid side chains is catalyzed by protein kinases and often induces significant conformational changes in the substrate protein. These changes can alter recognition by binding cognates and potentially have profound effects on protein activity [1]. Crucially, it is not only the phosphorylation site location, but also the stoichiometry of modification that determines the effect and extent of changes in protein function. If the biological pathways that these phosphoproteins comprise are to be modeled to help understand these networks and their interactions, it is essential that the proportion of these proteins that are modified be assessed, in addition to determining their total quantity. Although the identification of phosphorylation sites by mass spectrometry (MS) is becoming routine, this analysis is usually qualitative $[2,3]$. A number of studies have used the relative signal intensities of the phosphorylated peptide and the cognate nonphosphorylated peptide to infer phosphorylation stoichiometry $[2,4,5]$. However, recent evidence indicates that the introduction of a negatively charged phosphate group can alter mass spectrometric response factor to an unpredictable

Address reprint requests to Professor S. J. Gaskell, University of Manchester, Manchester Interdisciplinary Biocentre, John Garside Building, 131 Princess Street, Manchester M1 7DN, UK. E-mail: simon.gaskell@manchester.ac.uk degree, meaning that extent of peptide phosphorylation cannot be determined by simple comparison of these signal intensities [6, 7].

There are a number of alternative approaches that have been used to determine the stoichiometry of protein phosphorylation. Traditionally, this has involved assessing the amount of ${ }^{32} \mathrm{P}$ incorporation $[8,9]$, although expense and safety considerations have reduced the attractiveness of radioactive-based methods. Alternatively, mass spectrometric experiments can be performed to determine the exact amount of phosphorylated and nonphosphorylated peptides, thereby inferring the stoichiometry of phosphorylation, by addition of known amounts of synthetic stable isotope-labeled analogues of both variants [10, 11]. This approach requires the synthesis and purification of numerous stable isotope-labeled internal standard peptides in their phosphorylated and nonphosphorylated forms [12]. Elucidation of the stoichiometry of phosphorylation has also been demonstrated using a combination of stable isotope labeling and alkaline phosphatase treatment $[13,14]$. This approach requires the sample of interest to be divided into two aliquots: one is subjected to phosphatase treatment and subsequent derivatization (e.g., methyl esterification), whereas the second is subjected only to derivatization, using an isotopically distinct (esterification) reagent. Mass spectrometric analysis of the recombined mixture reveals light/heavy ratios differing from unity for those peptides incorporating a phosphorylation site; the extent of the discrep- 
ancy indicates stoichiometry [13, 14]. An alternative approach has been developed where labeling of proteins is performed before digestion, thus avoiding the variation that can be introduced due to phosphorylationdependent changes in the efficiency of proteolysis [15]. Nonetheless, all of these strategies require complete (or at least reproducible) derivatization and are compromised if enzymatic removal of the phosphate group is incomplete.

To overcome the potential differences in ionization efficiency of phosphorylated peptides, large-scale comparative studies of changes in the extent of specific protein phosphorylation has been achieved by the application of stable isotope labeling of amino acids in culture (SILAC), a method of differentially labeling numerous protein populations during cell culture [16, 17]. However, this approach provides no information on absolute levels of phosphorylated peptides. Steen et al. introduced a label-free approach to estimate changes in phosphorylation stoichiometry in a sample series; in addition, absolute stoichiometries were estimated based on the correlation of changes in the signals associated with phosphorylated tryptic peptides and their nonphosphorylated counterparts by [18]. The approach depends on the analysis of a sample series showing variations in phosphorylation stoichiometry.

Here, we describe a broadly applicable strategy that enables absolute quantification of proteins concurrently with a rigorous determination of the phosphorylation stoichiometry at specific sites. The strategy represents a development of our previously reported QconCAT method [19], in which absolute quantification of proteins is achieved using internal standards obtained via the expression of an artificial protein encoding a concatenation of multiple "signature" tryptic peptides [19, 20]. By using, for a single protein, a signature peptide that incorporates the phosphorylation site of interest and a second that incorporates no modification site, two quantitative values are obtained and the discrepancy defines the stoichiometry of phosphorylation (Scheme $\mathbf{1}$ ). In addition, we illustrate the use as internal standard of a stable isotope labeled analog of the nonphosphorylated protein. In both of these methods, quantification of stoichiometry is based upon the assumption that the diminution of signal associated with a nonmodified peptide is due to phosphorylation. We have assessed this assumption by comparing results before and after phosphatase treatment.

The applicability of these novel approaches is demonstrated by the analysis of sites of autophosphorylation on the catalytic domain of monopolar spindle 1 (Mps1) protein expressed in E. coli. Mps1 is a mitotic protein kinase required for the spindle assembly checkpoint (SAC) in many organisms including yeast, flies, zebrafish, frogs, and humans [4, 21]. Autophosphorylation of Mps1 is required for full activity in vitro with complex changes in the extent and location of phosphorylation known to be responsible for regulating protein kinase function $[4,21]$. We previously identified

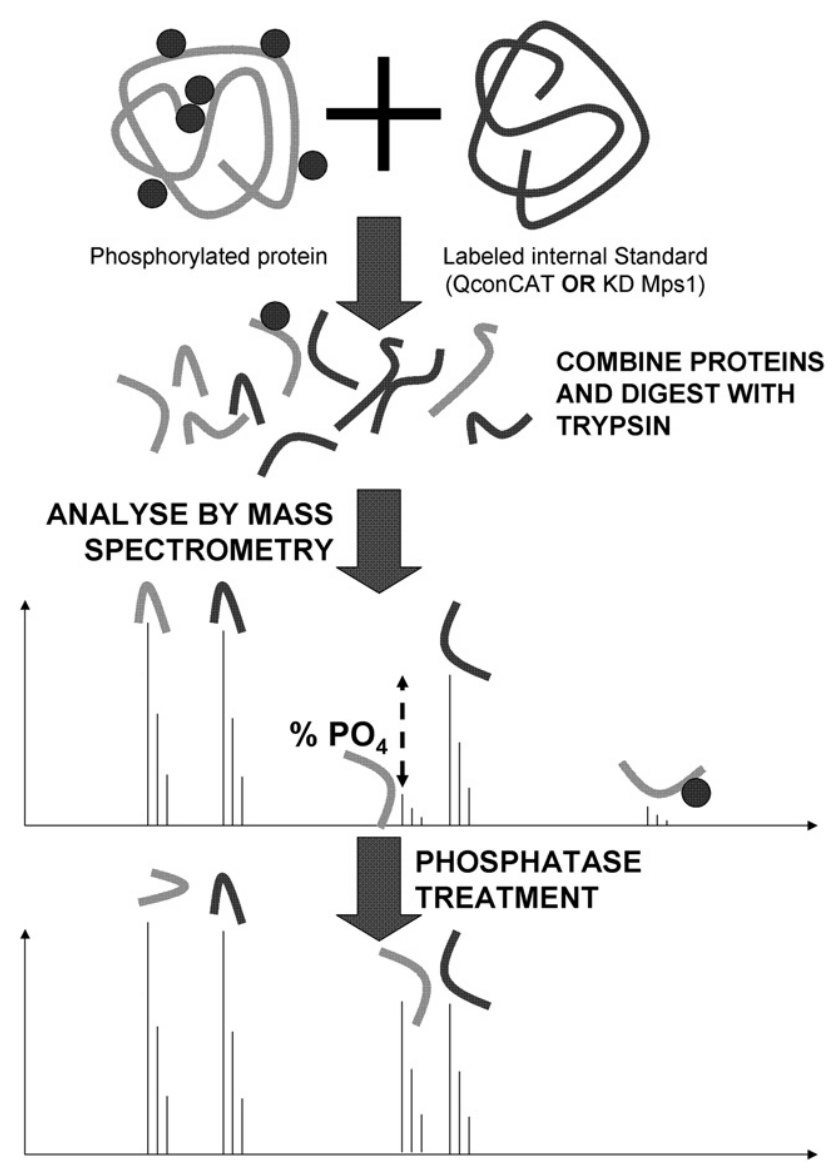

Scheme 1. Isotopically labeled internal standard protein (QconCAT or KD Mps1) is added to the phosphorylated protein of interest and digested with trypsin. Peptides incorporating a site of phosphorylation result show a reduced light/heavy ratio during MS analysis, in comparison with sequences not incorporating a phosphorylation site. The extent of the discrepancy reveals the stoichiometry of phosphorylation.

16 sites of autophosphorylation using liquid chromatography (LC)-MS/MS, incorporating the complementary fragmentation strategies of collision-induced dissociation and electron-transfer dissociation [3]. Twelve of the 16 sites identified are novel in vitro autophosphorylation sites, with two of these sites previously identified in vivo [3].

\section{Experimental}

\section{QconCAT Design and Expression}

Peptide sequences were selected for inclusion in the QconCAT based on previously obtained LC-ESI-MS/ MS data [3]. One peptide was included for each site of phosphorylation in addition to two peptides from a region that could not be phosphorylated (i.e., no serine, threonine, or tyrosine residues) or had never been identified as being phosphorylated. Due to the presence of missed cleavage peptides within the native protein during the initial experiments, flanking sequences either side of the tryptic peptide were incorporated into 
the design of the QconCAT. These consisted of between four and 13 amino acid residues $\mathrm{N}$-terminal to the Q-peptide. Following completion of the QconCAT design, the gene vector was generated (Entelechon $\mathrm{GmBH}$, Regensburg, Germany).

The QconCAT protein and the "kinase-dead" (KD) and, therefore, nonphosphorylated Mps1 protein [3] were expressed in the presence of $\left[{ }^{13} \mathrm{C}_{6}\right] \mathrm{R} / \mathrm{K}$ and purified as previously described [20].

\section{Tryptic Digestion}

To assess the stoichiometry of phosphorylation, a known quantity of $\left[{ }^{13} \mathrm{C}_{6}\right]-\mathrm{R} / \mathrm{K} \mathrm{KD}$ Mps1 or $\left[{ }^{13} \mathrm{C}_{6}\right]-\mathrm{R} / \mathrm{K}$ QconCAT was added to WT (wild type) Mps1 (highly purified from E. coli). Proteins were reduced with dithiothreitol at $5 \mathrm{mM}$ at $60{ }^{\circ} \mathrm{C}$ for $15 \mathrm{~min}$, alkylated with $20 \mathrm{mM}$ iodoacetamide at room temperature for 45 min, quenching excess iodoacetamide with dithiothreitol to a final concentration of $10 \mathrm{mM}$. Proteins were digested by addition of trypsin $(2 \%(w t / w t))$ at $37^{\circ} \mathrm{C}$ for $4 \mathrm{~h}$. An additional dose of trypsin [2\% (wt/wt)] was added and digestion was completed at $37^{\circ} \mathrm{C}$ for $18 \mathrm{~h}$.

\section{Peptide Desalting}

Peptide samples were acidified by addition of formic acid and desalted using $\mathrm{C}_{18}$ ZipTips (Millipore, Billerica, MA, USA), eluting with $80 \%$ (vol/vol) acetonitrile $0.1 \%$ (vol/vol) formic acid.

\section{Phosphatase Treatment}

Lambda phosphatase (1000 units) (New England Biolabs, Hertfordshire, UK) was added to $\sim 10$ pmol of digested phosphoprotein and was incubated at $30{ }^{\circ} \mathrm{C}$ for $2 \mathrm{~h}$ in $50 \mu \mathrm{L}$ of $50 \mathrm{mM}$ Tris- $\mathrm{HCl}, 100 \mathrm{mM} \mathrm{NaCl}, 0.1$ $\mathrm{mM}$ EGTA, $2 \mathrm{mM}$ dithiothreitol, and $20 \mathrm{mM} \mathrm{MnCl}_{2}$ at $\mathrm{pH}$ 7.9. Ten units of calf intestinal alkaline phosphatase (New England Biolabs, Hertfordshire, UK) were added to $\sim 10$ pmol of digested phosphoprotein. The mixture was incubated at $30^{\circ} \mathrm{C}$ for $1 \mathrm{~h}$ in $50 \mu \mathrm{L} 50 \mathrm{mM}$ Tris- $\mathrm{HCl}$,
$100 \mathrm{mM} \mathrm{NaCl}, 0.1 \mathrm{mM}$ EGTA, $1 \mathrm{mM}$ dithiothreitol, 20 $\mathrm{mM} \mathrm{MnCl} \mathrm{M}_{2}$, pH 7.5. Following phosphatase treatment, peptides were desalted (as described above) before mass spectrometric analysis.

\section{Mass Spectrometric Analysis}

Samples were analyzed by MALDI-TOF on an Ultraflex II (Bruker, Coventry, UK) before LC-ESI-MS. Approximately $100 \mathrm{fmol}$ of digested protein was co-crystallized with 2,5-dihydroxybenzoic acid matrix $(20 \mathrm{mg} / \mathrm{mL}$ in $50 \%$ (vol/vol) acetonitrile, $0.1 \%$ (vol/vol) formic acid). For all LC-ESI-MS experiments, the digestion mixture was acidified to $0.1 \%$ (vol/vol) formic acid, injected into an Ultimate 3000 capillary LC system (Dionex, Camberley, Surrey, UK) via a FAMOS autosampler, and separated using a $75 \mu \mathrm{m}$ reverse-phase capillary column (15 $\mathrm{cm})$ (LC Packings, Sunnyvale, CA, USA), at a flow rate of $200 \mathrm{~nL} / \mathrm{min}$, in-line with nano-electrospray source of a Q-TOF Global instrument (Waters, Manchester, UK). Where LC-ESI-MS analyses were used for quantification, ratios (light/heavy) were determined based on extracted ion chromatograms (XICs) produced for each peptide pair using QuanLynx (Waters). To ensure that the correct peptides were quantified, MS/MS raw data (from a separate LC-ESI-MS/MS analysis) were submitted to MASCOT, and searched against a database containing the QconCAT sequence for peptide identification.

\section{Results and Discussion}

\section{QconCAT Design}

A QconCAT protein was designed to incorporate peptide sequences that report on phosphorylated and nonphosphorylated tryptic peptides from five proteins including Mps1. The Mps1 phosphorylated sites were identified in previous qualitative analyses [3]. The design of this QconCAT construct enabled the simultaneous production of 40 peptides for the absolute quantification of the stoichiometry of 32 sites of phosphorylation in four proteins;

Table 1. List of peptides incorporated into the QconCAT for determination of the stoichiometry of Mps1 phosphorylation sites. The peptides highlighted in bold were incorporated for the absolute quantification of the total protein. The peptides underlined were incorporated as they had previously been identified as being phosphorylated (sites identified are indicated). Peptide GGVNDNEEGFFSAR was incorporated as a reference for quantification of the QconCAT

\begin{tabular}{|c|c|c|}
\hline Protein & Phosphorylation site(s) identified & Sequence \\
\hline Mps1 & - & GQTTKARFLYGENMPPODAEIGYRNSLROTNK \\
\hline Mps1 & - & AVERGAVPLEMLEIALRNLNLOKKOLLSEEEK \\
\hline Mps1 & S582 & YLNKLQQHSDKIIR \\
\hline Mps1 & S742 & MTYGKTPFQQIINOISK \\
\hline Mps1 & Y811, S821 \& S824 & GTTEEMKYVLGQLVGLNSPNSILK \\
\hline Mps1 & T564 & YAIKYVNLEEADNOTLDSYRNEIAYLNK \\
\hline Mps1 & T795 & CCLKDPKQRISIPELLAHPYVOIOTHPVNQMAK \\
\hline Mps1 & $\mathrm{S} 682 \& \mathrm{~T} 686$ & SVVKDSOVGTVNYMPPEAIK \\
\hline Mps1 & $\mathrm{T} 675$ \& $\mathrm{T} 676$ & GMLKLIDFGIANQMQPDTTSVVK \\
\hline Mps1 & S533 & SILKOIGSGGSSKVFOVLNEK \\
\hline Standard & - & GGVNDNEEGFFSAR \\
\hline
\end{tabular}


ten of these are sites are located on Mps1 (Table 1). The flanking sequences of these 40 tryptic peptides corresponded to their contexts in the target proteins, thus accounting for any potential missed cleavage (Table 1 ). Wherever possible, sequences were ordered in the QconCAT as in the native sequence. The QconCAT was expressed in E. coli grown in minimal medium supplemented with $\left[{ }^{13} \mathrm{C}_{6}\right]$-analogues of arginine and lysine.

We also produced a $\mathrm{His}_{6}$-tagged form of the Mps1 mutant, incorporating a D664A substitution (where amino acids are numbered based on the full length sequence) in the catalytic domain, rendering the kinase inactive ("kinase dead", KD). The expressed proteins were purified by virtue of their $\mathrm{His}_{6}$-tags and KD Mps1 was quantified using a standard Bradford protein assay. The QconCAT protein was quantified by MS using an additional tryptic peptide unrelated to the proteins of interest and incorporated for this purpose (GVNDNEEGFFSAR). Specifically, a digest of the QconCAT protein was co-analyzed with a known quantity of chemically synthesized GVNDNEEGF*FSAR (where *F indicates $\left[{ }^{13} \mathrm{C}_{9},{ }^{15} \mathrm{~N}_{1}\right]-\mathrm{F}$ ) to allow determination of the quantity of protein. A known quantity of chemically synthesized GVNDNEEGF*FSAR was added with each use of the QconCAT for quantitative analysis of WT Mps1 protein by LC-ESI-MS. It is important to note here that the sequence within the QconCAT contained an additional glycine residue at the $\mathrm{N}$-terminus. This has previously been shown to have no effect on the response factor when analyzed by electrospray ionization (ESI) using a QTOF mass spectrometer [22]. This strategy was employed to allow the absolute quantification of numerous QconCATs simultaneously without the requirement for differential isotopic labeling of each QconCAT protein. Using this approach, the concentration of the QconCAT was determined as $130 \pm 19$ $\mathrm{fmol} / \mu \mathrm{l}$ (coefficient of variation (CV) of $15 \%, n=5$ preparations). The QconCAT protein was absolutely quantified within the same experiment as used to determine the stoichiometry of phosphorylation, allowing also the absolute quantification of WT Mps1 as $148 \pm 38 \mathrm{fmol} / \mu \mathrm{L}$ ( $n=4$ preparations). Though the QconCAT was designed for the study of several proteins, only analyses of Mps1 are reported here, allowing a focus on the alternative use of the stable isotopelabeled nonphosphorylated Mps1.

\section{Determination of the Stoichiometry of Phosphorylation}

The stable isotope labeled KD Mps1 or QconCAT was added in known quantities to bacterially expressed autophosphorylated Mps1 before tryptic digestion. The digest was analyzed by matrix-assisted laser desorption ionization (MALDI)-MS and LC-ESI MS. Two or more reference peptides from unmodified regions of Mps1 were used to identify the ratios (unlabeled/labeled: $(\mathrm{L} / \mathrm{H})_{\text {ref }}$ ) in which the two proteins (Mps1/KD Mps1 or
Mps1/QconCAT) were mixed, thus enabling absolute quantification of Mps1. This use of multiple reference peptides from nonphosphorylated regions and assessment of any variation between the $\mathrm{L} / \mathrm{H}$ ratios for these peptides provides evidence for any unexpected modification of these sequences. Isotopic ratios $\left((\mathrm{L} / \mathrm{H})_{\bmod }\right)$ were also determined for each of the tryptic peptides incorporating phosphorylation sites, and the phosphorylation stoichiometry calculated in each case using the following equation:

$$
\begin{aligned}
& \text { Stoichiometry }(\%) \\
& \qquad=100-\left[100 \times\left((\mathrm{L} / \mathrm{H})_{\bmod } /(\mathrm{L} / \mathrm{H})_{\mathrm{ref}}\right)\right]
\end{aligned}
$$

To substantiate the effectiveness of tryptic digestion, we co-analyzed isotope-labeled QconCAT and unlabeled KD Mps 1 and demonstrated consistent $\mathrm{L} / \mathrm{H}$ ratios for all signature peptides $(0.84 \pm 0.13$; mean $\pm \mathrm{CV}, n=8$, the number of peptides analyzed). These experiments were repeated six times resulting in instrumental CVs of less than 3\% for each peptide measured. Additionally, the efficiency of tryptic digestion was also assessed. A repeated dose of trypsin was added after $4 \mathrm{~h}$ and then again after $18 \mathrm{~h}$. The $\mathrm{L} / \mathrm{H}$ ratio of $\left[{ }^{13} \mathrm{C}_{6}\right]-\mathrm{R} / \mathrm{K}$ labeled QconCAT or KD Mps1 to unlabelled wild-type Mps1 was monitored and found not to change after the second dose of trypsin (data not shown), thus indicating that tryptic digestion had been completed as efficiently as possible.

\section{Use of $\left[{ }^{13} C_{6}\right]-R / K$ labeled $K D$ Mps1 for Phosphorylation Determination}

Figure 1a shows four regions of the MALDI-TOF mass spectrum, corresponding to isotopic variants of one peptide not incorporating a modification site (SIDPWER) and three peptides incorporating sites of phosphorylation (TPFQQIINQISK, YVNLEEADNQTLDSYR, and DSQVGTVNYMPPEAIK). The $\mathrm{L} / \mathrm{H}$ ratios were determined from the summed areas of the first three peaks in each isotope cluster, enabling calculation of the stoichiometries of phosphorylation as $26 \% \pm 4 \%, 53 \% \pm$ $1 \%$, and $80 \% \pm 1 \%$ for $n=6$ (where $n=$ number of instrumental repeats), respectively. For the peptide DSQVGTVNYMPPEAIK, prior qualitative analyses [3] indicated the presence of two phosphopeptide forms: DSQVGPTVNYMPPEAIK and DpSQVGpTVNYMPPEAIK. The quantitative analyses reported here demonstrate that this sequence is either mono- or bis-phosphorylated to $80 \%$. As T686 is phosphorylated in both modified forms of this peptide, these data indicate that the amino acid T686 is phosphorylated with a stoichiometry of $80 \%$. A similar situation arises with the peptide YVLGQLVGLNSPNSILK, on which three sites of phosphorylation have previously been identified. How- 

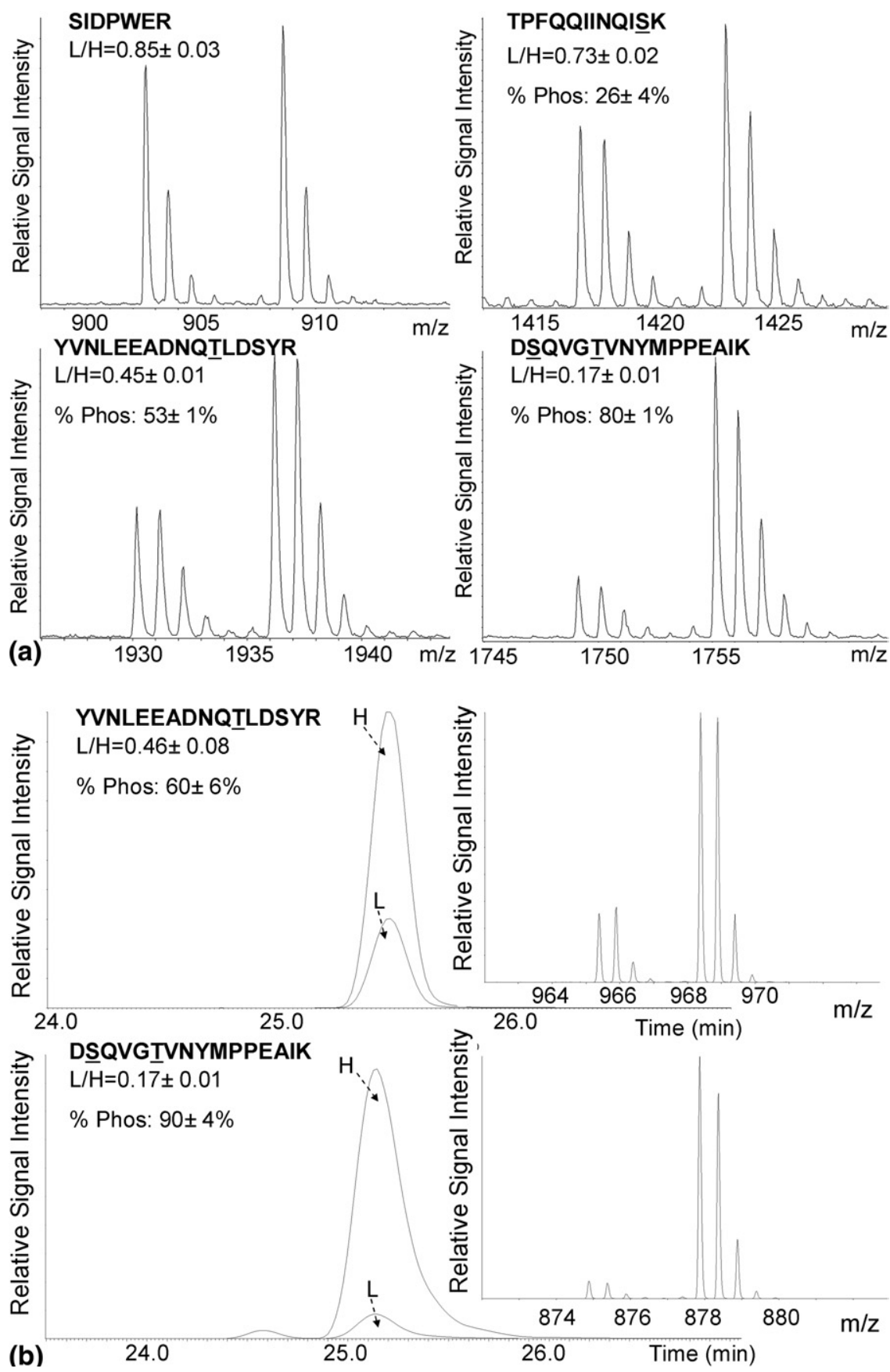

Figure 1. Quantification of the stoichiometry of phosphorylation using isotopically labeled KD Mps1 analyzed by MALDI-TOF and LC-ESI-MS. (a) Quantification following MALDI-TOF analysis was performed using the summed areas of the isotopic variants of each peptide. The stoichiometry of phosphorylation was calculated as described in the text. Peptide SIDPWER is a reference nonphosphorylated peptide, whilst the other peptides exhibit a decreased $\mathrm{L} / \mathrm{H}$ ratio indicative of modification. Underlined residues have been identified as phosphorylated. These data are representative of $n=6$ analyses. (b) Extracted ion chromatogram (XIC) of the ion corresponding to the doubly protonated species of the labeled (H) (red line) and unlabeled (L) (grey line) forms of YVNLEEADNQTLDSYR (top) and DSQVGTVNYMPPEAIK (bottom). Partial mass spectra corresponding to these XICs are inset.

ever, as the tandem MS data indicate that S824 (YVLGQLVGLNSPNpSILK) is modified in all phosphoforms of this peptide, the stoichiometry of phosphorylation of S824 can be determined as 93\% whilst the stoichiometry of sites S821 and Y811 remain unknown.
Comparison of MALDI-MS and LC-ESI-MS Quantification

Figure $1 \mathrm{~b}$ exemplifies LC-ESI-MS data for two of these same peptides, YVNLEEADNQTLDSYR and 
DSQVGTVNYMPPEAIK, in the form of extracted ion chromatograms (XICs) for each peptide pair; L/H ratios were determined from the areas under each chromatographic peak. In each case, estimates of $(\mathrm{L} / \mathrm{H})_{\text {ref }}$ using the $\left[{ }^{13} \mathrm{C}_{6}\right]-\mathrm{R} / \mathrm{K}$ labeled KD Mps1, were based on determinations of four nonphosphorylated peptides, including SIDPWER; mean estimates were obtained with a coefficient of variation of $9 \%$ and $13 \%$ from MALDITOF MS and LC-ESI MS analyses, respectively. When using the $\left[{ }^{13} \mathrm{C}_{6}\right]-\mathrm{R} / \mathrm{K}$ labeled QconCAT, four nonphosphorylated peptides were used, including peptides within the flanking regions (Table 1). Mps1 peptides YVNLEEADNQpTLDSYR and DSQVGPTVNYMPPEAIK were previously identified to be phosphorylated on T564 and T686, respectively. Using the isotope-labeled form of KD-Mps1, the stoichiometry of phosphorylation was calculated to be $60 \%$ and $90 \%$ for these two peptides. Table 2 shows a comparison of stoichiometry data for eight phosphorylation sites, five of which were determined by both MALDI-TOF MS and LC/ESI-MS. However, MALDI-TOF MS could not be used to determine the stoichiometry of phosphorylation for three peptides due to the presence of overlapping peptide ions which prevented accurate calculation of peak areas; the two MS methods otherwise showed good agreement within the limits of the observed precision. Generally, the calculated stoichiometry of phosphorylation was marginally lower using the MALDI-TOF MS data, which can be explained by the lack of peptide chromatographic separation of the MALDI-TOF analyzed mixture, resulting in the presence of overlapping species. The method incorporating coupled chromatographic separation is therefore generally to be preferred, as this enables separation and thus accurate quantification of peptide ions of similar $\mathrm{m} / \mathrm{z}$. An example of this is the peptide TLYEHYSGGESHNSSSSK at $\mathrm{m} / \mathrm{z}$ 1969.85, where significant overlapping signals pre- vented analysis by MALDI-TOF MS, but the extra dimension of separation afforded by reversed-phase chromatography permitted quantification (Figure 2). Where no overlapping peptide ion signals are present, the correlation between the stoichiometry of phosphorylation obtained by MALDI-TOF MS analyses is in accordance with LC-ESI-MS quantification. In all experiments, the MALDI-TOF MS stoichiometry of phosphorylation calculations are within $11 \%$ of that for the LC-ESI-MS analyses. Significantly, it is important to note that the instrumental variation for both the MALDI-TOF MS and LC-ESI MS analyses were consistently below $5 \%$.

\section{Comparison of Determination of Phosphorylation Stoichiometry Using $\left[{ }^{13} C_{6}\right]-R / K$ Labeled QconCAT and KD Mps1}

The stoichiometry data obtained using the $\left[{ }^{13} \mathrm{C}_{6}\right] \mathrm{R} / \mathrm{K}$ labeled KD Mps1 and the QconCAT were also compared (Figure 3 and Table 3) and demonstrated agreement within $10 \%$. Technical experimental variation was assessed $(n=6$, where sample preparation and mass spectrometric analyses were independently carried out for each replicate). At extreme $\mathrm{L} / \mathrm{H}$ ratios, where either the unlabeled or labeled ion is of relatively low signal intensity, notably for the peptide LIDFGIANQMQPDTTSVVK, the $\mathrm{CVs}$ were higher than those where the $\mathrm{L} / \mathrm{H}$ ratios were closer to unity (Table 3). The stoichiometry of phosphorylation was calculated for fewer peptides using the QconCAT than the kinase dead Mps1 catalytic domain as only selective Mps1 peptides were incorporated into the QconCAT design (comparison of Tables 2 and 3).

Phosphorylation sites T676 (LIDFGIANQMQPDTpTSVVK) and T686 (DSQVGPTVNYMPPEAIK) have been identified as sites crucial for the full catalytic activity of

Table 2. Comparison of the stoichiometry of phosphorylation calculated using $\left[{ }^{13} \mathrm{C}_{6}\right]-\mathrm{R} / \mathrm{K}$ labeled KD Mps1 analyzed by MALDI-ToF MS and LC-ESI-MS. Each mass spectrometric experiment was performed six times to indicate the reproducibility of instrumental analysis for the percentage stoichiometry; the mean stoichiometry and coefficient of variation (CV) are included for each peptide

\begin{tabular}{|c|c|c|c|c|}
\hline \multirow[b]{2}{*}{ Workflow } & \multirow[b]{2}{*}{ Peptide sequence } & \multirow[b]{2}{*}{$m / z$} & \multicolumn{2}{|c|}{ Phosphorylation determination } \\
\hline & & & $\%$ Stoichiometry & $\mathrm{CV}(\%)$ \\
\hline LC-ESI-MS & ISIPELLAHPYVQIOTHPVNOMAK & 682.67 & 66 & 13.1 \\
\hline MALDI-MS & ISIPELLAHPYVQIOTHPVNOMAK & 2727.55 & 63 & 8.3 \\
\hline LC-ESI-MS & TPFQQIINQISK & 708.92 & 34 & 30.5 \\
\hline MALDI-MS & TPFQQIINQISK & 1416.85 & 25 & 16.2 \\
\hline LC-ESI-MS & DSOVGTVNYMPPEAIK & 874.98 & 88 & 4.9 \\
\hline MALDI-MS & DSQVGTVNYMPPEAIK & 1748.94 & 80 & 1.4 \\
\hline LC-ESI-MS & YVLGOLVGLNSPNSILK & 908.00 & 95 & 2.0 \\
\hline MALDI-MS & YVLGQLVGLNSPNSILK & 1815.20 & 89 & 1.1 \\
\hline LC-ESI-MS & YVNLEEADNOTLDSYR & 965.50 & 60 & 9.5 \\
\hline MALDI-MS & YVNLEEADNQTLDSYR & 1929.98 & 53 & 2.5 \\
\hline LC-ESI-MS & MASSSANECISVK & 692.36 & 82 & 6.8 \\
\hline MALDI-MS & MASSSANECISVK & 1383.62 & - & - \\
\hline LC-ESI-MS & QHMDSPDLGTDDDDK & 563.58 & 66 & 29.7 \\
\hline MALDI-MS & QHMDSPDLGTDDDDK & 1688.67 & - & - \\
\hline LC-ESI-MS & TLYEHYSGGESHNSSSSK & 657.29 & 99 & 0.4 \\
\hline MALDI-MS & TLYEHYSGGESHNSSSSK & 1969.85 & - & - \\
\hline
\end{tabular}




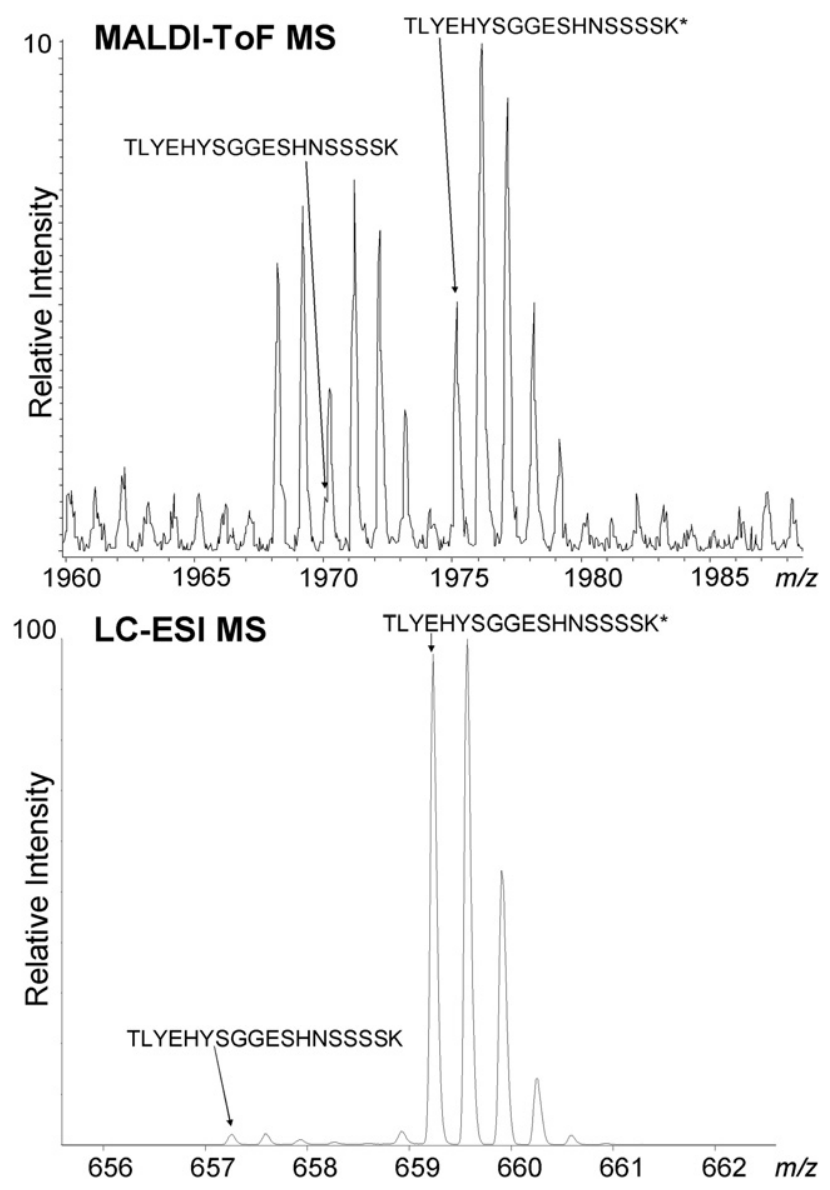

Figure 2. Mass spectra showing peptide TLYEHYSGGESHNSSSSK and its labeled counterpart TLYEHYSGGESHNSSSSK* analyzed by MALDI-TOF MS (top panel) and LC-ESI MS (bottom panel). The monoisotopic masses of the labeled and unlabeled forms of the peptide are indicated with arrows in both panels.

the Mps1 protein kinase [21]. Our analyses failed to detect any nonphosphorylated T676, while the stoichiometry of phosphorylation of T686 was $95 \% \pm 1 \%$, indicating that both sites are highly modified.

\section{Assessment of the Influence of Missed Cleavages}

All quantitative proteome analyses that employ peptide internal standards (however derived) incorporate the assumption that tryptic hydrolysis is fully effective; this assumption must be tested. In the present work, tryptic digestion of the phosphorylated protein may be differentially affected by the phosphorylation. The significance of missed cleavage was reduced by performing digestion in two stages, corresponding to separate additions of trypsin, separated by $4 \mathrm{~h}$, with a total digestion time of $22 \mathrm{~h}$. Nevertheless, we identified one miss-cleaved peptide consistently in our experiments, namely YVNLEEADNQTLDSYRNEIAYLNK, the full sequence of which was incorporated in the QconCAT protein. We compared the $\mathrm{L} / \mathrm{H}$ ratios of the fully cleaved peptide YVNLEEADNQTLDSYR and YVNLEEADNQTLDSYRNEIAYLNK within the QconCAT experiments (Figure 4a and b) and the KD Mps1 experiments (Figure $4 \mathrm{c}$ and $\mathrm{d}$ ).

Within the QconCAT experiment, the $\mathrm{L} / \mathrm{H}$ ratio of YVNLEEADNQTLDSYR was $1.62 \pm 0.06(n=6$ replicate LC-MS analyses) whilst the $\mathrm{L} / \mathrm{H}$ ratio for YVNLEEADNQTLDSYRNEIAYLNK was $1.33 \pm 0.13$ $(n=6)$ (Figure $4 \mathrm{a}$ and $\mathrm{b}$, respectively). The $\mathrm{L} / \mathrm{H}$ ratios for the fully cleaved and the miss-cleaved peptide were $0.85 \pm 0.01$ and $1.00 \pm 0.03$ within the KD Mps1 experiments (Figure $4 \mathrm{c}$ and $\mathrm{d}$, respectively). This indicates a modest difference of $15 \%$ between the two sets of peptides (fully cleaved and miss-cleaved) within both the QconCAT and the KD Mps1 experiments. The difference of $15 \%$ in the KD Mps1 could indicate that due to the presence of the phosphate these two populations of peptides are differentially represented. However, the difference in $\mathrm{L} / \mathrm{H}$ ratio in the QconCAT experiment could be due to the reduced signal-to-noise of the miss cleaved peptide, which would give rise to increased variation. This reduced presence of the misscleaved peptide could be due to the significantly different conformation of the QconCAT protein compared to the native Mps1 structure. However, although clearly a phosphate group has the potential to induce missed cleavage it does not impair the determination of phosphorylation upon this peptide.

\section{Assessment of Artifactual Modification of Tryptic Peptides}

The presence of artifactual modification can skew quantitative determinations if the extent of modification differs between phosphorylated peptide and internal standard. In the current experiments, a minor extent (less than 5\%) of methionine oxidation was observed; in these instances, the observed $\mathrm{L} / \mathrm{H}$ ratios for the modified peptide pairs were in agreement with those observed for the unmodified peptides.

\section{Analyses After Phosphatase Treatment}

If the differences in $\mathrm{L} / \mathrm{H}$ ratio between peptides incorporating a site of phosphorylation and those corresponding to unmodified sequences are indeed entirely attributable to phosphorylation, then prior treatment with a phosphatase enzyme should in principle result in the subsequent recording of a single, common $\mathrm{L} / \mathrm{H}$ ratio. In practice, this will be so only if phosphate hydrolysis is complete. In the present work, additional quantitative analyses were performed on wild type Mps1 after treatment with either alkaline or lambda phosphatase, using the labeled QconCAT or KD Mps1 as internal standard (Figure 5). In five of the eight peptides for which we report data, phosphatase treatment restored the $\mathrm{L} / \mathrm{H}$ ratio to $0.9-1.0$ of that observed for the reference peptides (data not shown). For DSQVGTVNYMPPEAIK (which contains T686), phos- 

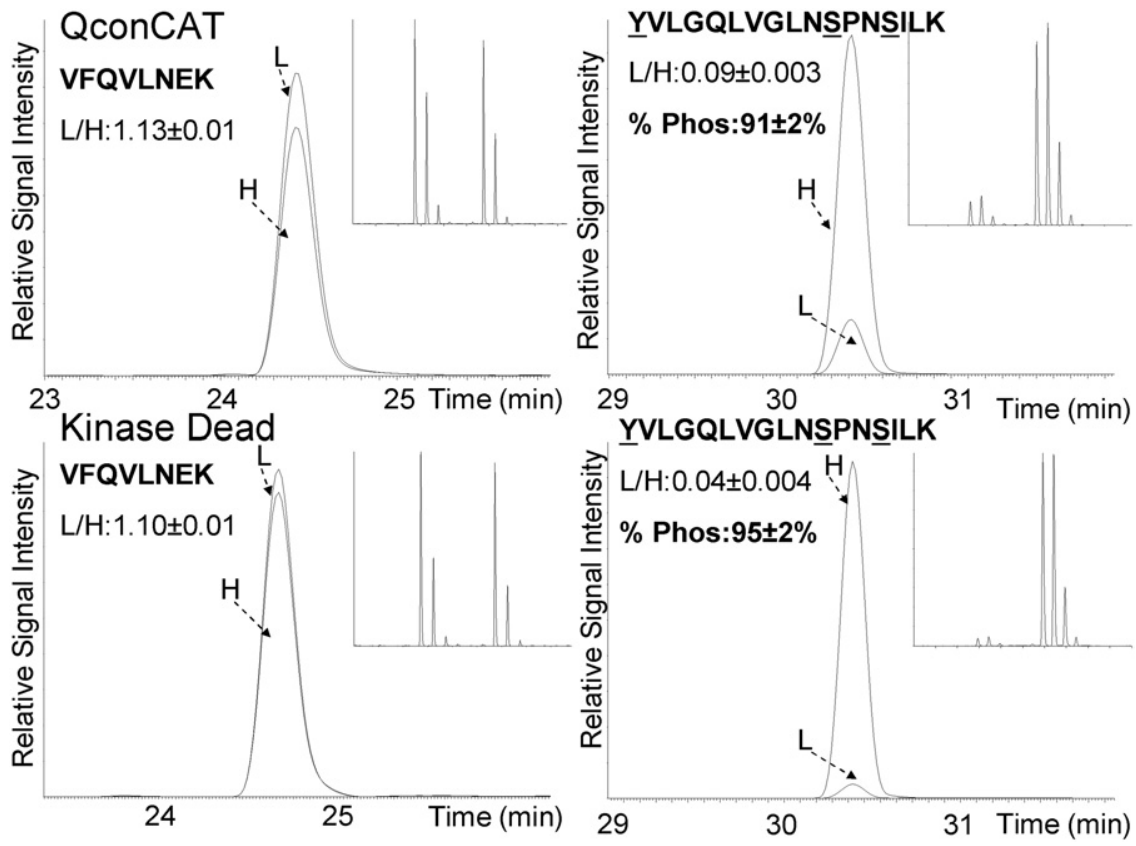

Figure 3. Absolute quantification of phosphopeptide stoichiometry using kinase dead internal standard protein and the QconCAT. XICs and mass spectra (inset) of reference peptide VFQVLNEK $\mathrm{L} / \mathrm{H}$ pair and phosphopeptide YVLGQLVGLNSPNSILK L/H pair. Underlined residues have been identified as phosphorylated.

phatase treatment increased the $\mathrm{L} / \mathrm{H}$ ratio from $0.06 \pm$ 0.002 to $1.03 \pm 0.01$ ( $n=6$ replicate LC-MS analyses) after alkaline phosphatase treatment, compared with $2.43 \pm 0.38(n=3$, the number of peptides analyzed) for the reference peptides, suggesting that phosphate hydrolysis was $45 \%$ complete for this peptide. Evaluation of the LC-MS data for the sample after phosphatase treatment, by examination of the extracted ion chromatogram corresponding to the phosphorylated peptide, failed to detect this component (Figure 5), but this may be attributable to limited detectability for this phosphopeptide. Equivalent observations with alkaline and $\lambda$ phosphatase was also made for both
LIDFGIANQMQPDTTSVVK and YVLGQLVGLNSPNSILK. These findings would be consistent with the presence of modifications other than phosphorylation, although other modifications are unlikely to occur during bacterial protein expression. Close inspection of the mass spectra revealed that the extents of methionine oxidation and deamidation for these peptides were not significantly altered after phosphatase treatment, which could potentially account for the differences observed. A more likely explanation, therefore, is that dephosphorylation is incomplete in these highly modified peptides, reinforcing the notion that calculations of stoichiometry based on comparative analyses before

Table 3. The calculated phosphorylation stoichiometry for each phosphorylated peptide, analyzed by LC-ESI-MS. The CV values indicate the reproducibility evident from analyses of multiple $(n=6)$ independent samples. Quantification could not be performed for LIDFGIANQMQPDTTSVVK using the kinase dead construct due to the insertion of the single point mutation (D664A) in KD Mps 1

\begin{tabular}{|c|c|c|c|c|c|}
\hline \multirow[b]{2}{*}{ Workflow } & \multirow[b]{2}{*}{ Peptide sequence } & \multirow[b]{2}{*}{$m / z$} & \multicolumn{2}{|c|}{$\mathrm{L} / \mathrm{H}$ ratio } & \multirow[b]{2}{*}{$\%$ Stoichiometry } \\
\hline & & & Mean & CV (\%) & \\
\hline Kinase dead & ISIPELLAHPYVQIOTHPVNOMAK & 682.67 & 0.493 & 6.3 & 58 \\
\hline QconCAT & ISIPELLAHPYVQIQTHPVNOMAK & 682.66 & 0.335 & 0.6 & 66 \\
\hline Kinase dead & TPFQQIINQISK & 708.92 & 0.600 & 3.4 & 39 \\
\hline QconCAT & TPFQQIINQISK & 708.81 & 0.606 & 9.3 & 45 \\
\hline Kinase dead & DSOVGTVNYMPPEAIK & 874.98 & 0.111 & 14.8 & 88 \\
\hline QconCAT & DSOVGTVNYMPPEAIK & 874.90 & 0.168 & 3.5 & 95 \\
\hline Kinase dead & YVLGQLVGLNSPNSILK & 908.00 & 0.041 & 10.6 & 95 \\
\hline QconCAT & YVLGQLVGLNSPNSILK & 907.95 & 0.121 & 8.8 & 91 \\
\hline Kinase dead & YVNLEEADNOTLDSYR & 965.50 & 0.404 & 24.1 & 56 \\
\hline QconCAT & YVNLEEADNQTLDSYR & 965.33 & 0.463 & 16.6 & 60 \\
\hline Kinase dead & LIDFGIANQMQPDTTSVVK & - & - & - & - \\
\hline QconCAT & LIDFGIANOMOPDTTSVVK & 1038.95 & 0.002 & 94.7 & 100 \\
\hline
\end{tabular}



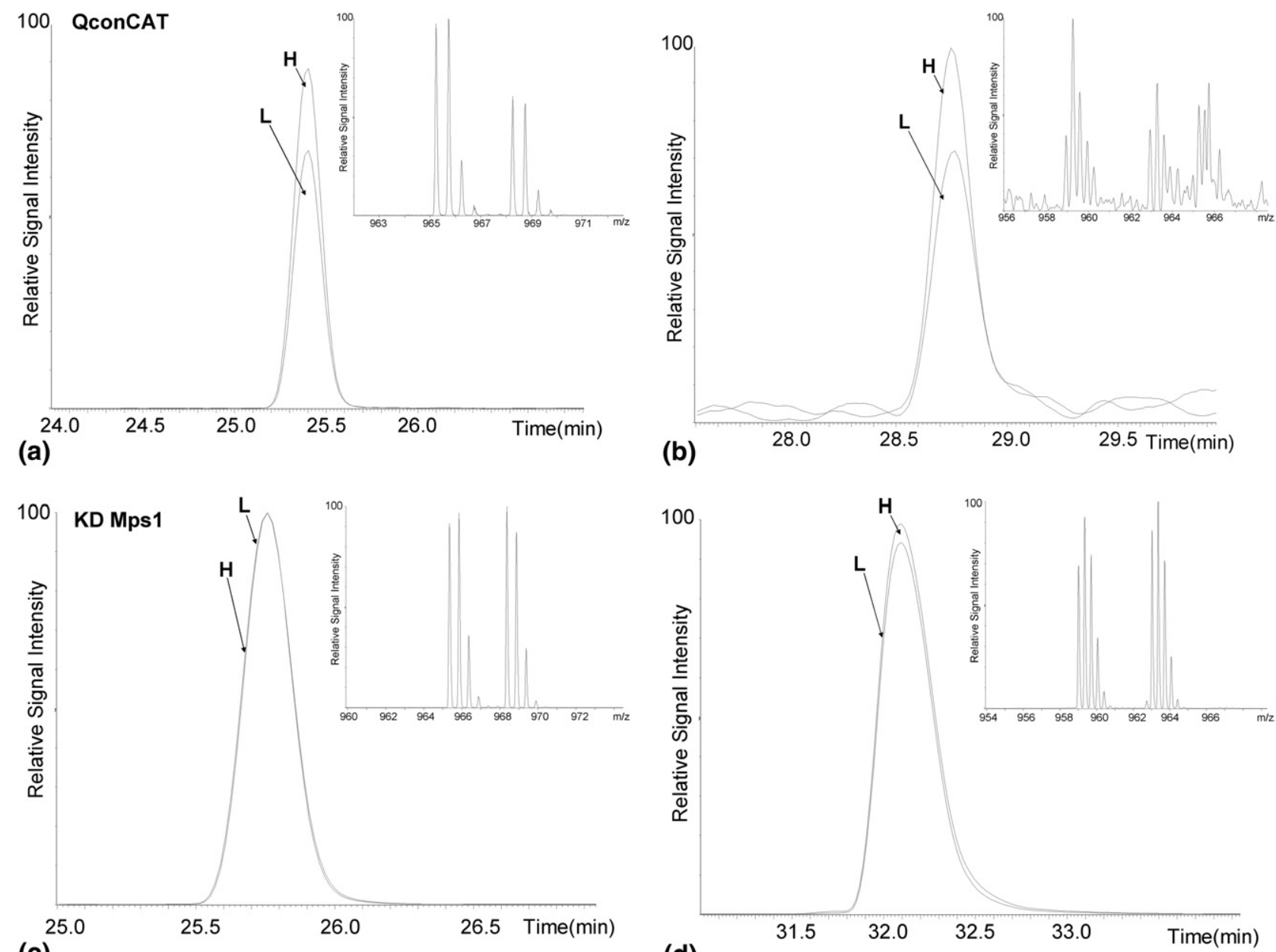

(c)

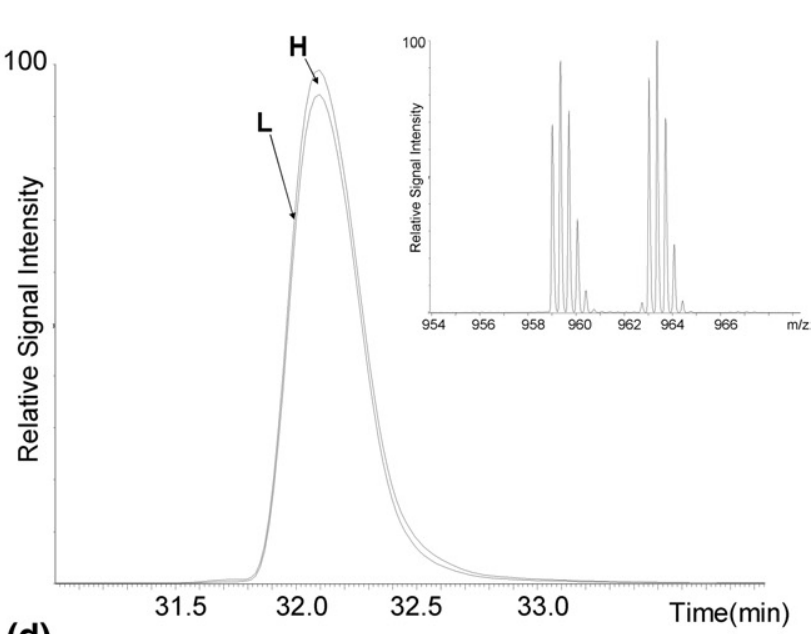

\begin{abstract}
Figure 4. LC-ESI-MS mass spectra and extracted ion chromatograms (XICs) of miss-cleaved peptide YVNLEEADNQTLDSYRNEIAYLNK and the fully cleaved peptide YVNLEEADNQTLDSYR and their corresponding labeled QconCAT or KD Mps1 masses. (a) XICs of YVNLEEADNQTLDSYR and its $\left[{ }^{13} \mathrm{C}_{6}\right]-\mathrm{R} / \mathrm{K}$ labeled QconCAT counterpart. (b) XICs of YVNLEEADNQTLDSYRNEIAYLNK and its $\left[{ }^{13} \mathrm{C}_{6}\right]-\mathrm{R} / \mathrm{K}$ labeled QconCAT counterpart. (c) XICs of YVNLEEADNQTLDSYR and its $\left[{ }^{13} \mathrm{C}_{6}\right]-\mathrm{R} / \mathrm{K}$ labeled KD Mps1 counterpart. (d) XICs of YVNLEEADNQTLDSYRNEIAYLNK and its $\left[{ }^{13} \mathrm{C}_{6}\right]-\mathrm{R} / \mathrm{K}$ labeled KD Mps1 counterpart. In all XICs, L refers to the unlabeled peptide and $\mathrm{H}$ to the labeled peptide.
\end{abstract}

and after phosphatase treatment have a risk of significant inaccuracy.

\section{Conclusions}

Stable isotope-labeled QconCAT and kinase dead protein have been utilized to determine the stoichiometry of phosphorylation and the absolute amounts of the catalytic domain of Mps1. Assessment by MALDI-TOF MS and LC-ESI MS for the quantitative analysis of phosphopeptide stoichiometry revealed a close agreement in the absence of overlapping ion signals. Phosphatase treatment (both alkaline and $\lambda$ phosphatase) confirmed the presence of phosphorylation on the peptides selected. However, although the phosphopeptides could not be observed after enzymatic treatment (presumably due to issues associated with their ionization and/or detection), this method could not be used for accurate determination of phosphopeptide levels due to incomplete phosphate removal. This should be kept in mind when using a phosphatase treatment strategy for calculating the stoichiometry of phosphorylation. The QconCAT method provides a rigorous method for concomitant absolute quantification of target proteins and determination of the stoichiometry of phosphorylation at known sites. In principle, the application of this approach can be extended to the quantification of other post-translational modifications, assuming prior qualitative characterization. During preparation of this manuscript, related strategies were described for calculating the stoichiometry of phosphorylation by Blair and coworkers and by Steen and coworkers, using isotope-labeled peptide and intact protein internal standards, respectively [11, 23]. The QconCAT approach presented here, however, is uniquely capable of determining the absolute amounts of multiple proteins and 


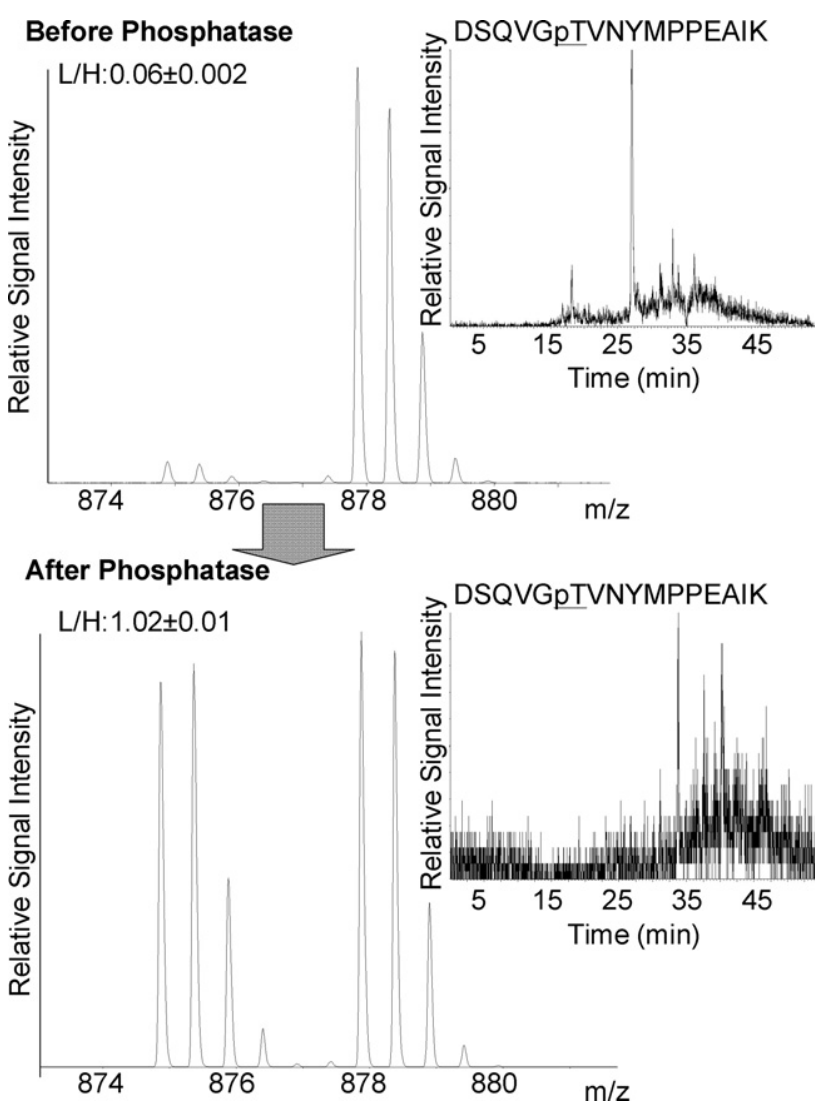

Figure 5. LC-ESI-MS mass spectra of DSQVGTVNYMPPEAIK $\mathrm{L} / \mathrm{H}$ peptide pair before (top panel) and after (bottom panel) phosphatase treatment. XICs of the singly phosphorylated peptide DSQVGPTVNYMPPEAIK are inset in top and bottom panels.

the stoichiometry of their modifications in a single experiment. Furthermore, whilst the data reported in this paper relate to multiple sites of phosphorylation in a single protein, the QconCAT was designed the enable the study of multiple proteins, exemplifying a general feature of this approach.

\section{Acknowledgments}

The authors acknowledge support for this work by grants from the Engineering and Physical Sciences Research Council to S.J.G. and R.J.B. (reference EP/D013615/1) and the Biotechnology and Biological Sciences Research Council to S.J.G. and R.J.B. (reference BB/D013615/1). C.E.E. is supported by a Royal Society Dorothy Hodgkin Fellowship. P.A.E is supported by grants from Yorkshire Cancer Research, Cancer Research UK, and the Medical Research Council.

\section{References}

1. Seet, B. T.; Dikic, I.; Zhou, M. M.; Pawson, T. Reading Protein Modifications with Interaction Domains. Nat. Rev. Mol. Cell. Biol. 2006, 7, $473-483$.
2. Haydon, C. E.; Eyers, P. A.; Aveline-Wolf, L. D.; Resing, K. A.; Maller, J. L.; Ahn, N. G. Identification of Novel Phosphorylation Sites on Xenopus laevis Aurora A and Analysis of Phosphopeptide Enrichment by Immobilized Metal-affinity Chromatography. Mol. Cell. Proteom. 2003, 2, 1055-1067.

3. Tyler, R. K.; Chu, M. L. H.; Johnson, H.; McKenzie, E. A.; Gaskell, S. J.; Eyers, P. A. Phosphoregulation of Human Mps1 Kinase. Biochem. J. 2009, 417, 173-181.

4. Mattison, C. P.; Old, W. M.; Steiner, E.; Huneycutt, B. J.; Resing, K. A.; Ahn, N. G.; Winey, M. Mps1 Activation Loop Autophosphorylation Enhances Kinase Activity. J. Biol. Chem. 2007, 282, 30553-30561.

5. Ruse, C. I.; Willard, B.; Jin, J. P.; Haas, T.; Kinter, M.; Bond, M. Quantitative Dynamics of Site-Specific Protein Phosphorylation Determined Using Liquid Chromatography Electrospray Ionization Mass Spectrometry. Anal. Chem. 2002, 74, 1658-1664.

6. Steen, H.; Jebanathirajah, J. A.; Rush, J.; Morrice, N.; Kirschner, M. W. Phosphorylation Analysis by Mass Spectrometry: Myths, Facts, and the Consequences for Qualitative and Quantitative Measurements. Mol. Cell. Proteom. 2006, 5, 172-181.

7. Gropengiesser, J.; Varadarajan, B. T.; Stephanowitz, H.; Krause, E. The Relative Influence of Phosphorylation and Methylation on Responsiveness of Peptides to MALDI and ESI Mass Spectrometry. J. Mass Spectrom. 2009, 44, 821-831.

8. Brábek, J.; Hanks, S. Assaying Protein Kinase Activity. Methods Mol. Biol. 2004, 284, 79-90.

9. Haydon, C. E.; Watt, P. W.; Morrice, N.; Knebel, A.; Gaestel, M.; Cohen, P. Identification of a Phosphorylation Site on Skeletal Muscle Myosin Light Chain Kinase That Becomes Phosphorylated during Muscle Contraction. Arch. Biochem. Biophys. 2002, 397, 224-231.

10. Gerber, S. A.; Rush, J.; Stemman, O.; Kirschner, M. W.; Gygi, S. P. Absolute Quantification of Proteins and Phosphoproteins from Cell Lysates by Tandem MS. Proc. Natl. Acad. Sci. U.S.A. 2003, 100, 69406945.

11. Ciccimaro, E.; Hanks, S. K.; Yu, K. H.; Blair, I. A. Absolute Quantification of Phosphorylation on the Kinase Activation Loop of Cellular Focal Adhesion Kinase by Stable Isotope Dilution Liquid Chromatography/ Mass Spectrometry. Anal. Chem. 2009, 81, 3304-3313.

12. Mayya, V.; Rezual, K.; Wu, L.; Fong, M. B.; Han, D. K. Absolute Quantification of Multisite Phosphorylation by Selective Reaction Monitoring Mass Spectrometry: Determination of Inhibitory Phosphorylation Status of Cyclin-Dependent Kinases. Mol. Cell. Proteom. 2006, 5, $1146-1157$.

13. Zhang, X.; Jin, Q.; Carr, S.; Annan, R. N-Terminal Peptide Labeling Strategy for Incorporation of Isotopic Tags: A Method for the Determination of Site-Specific Absolute Phosphorylation Stoichiometry. Rapid Commun. Mass Spectrom. 2002, 16, 2325-2332.

14. Hegeman, A. D.; Harms, A. C.: Sussman, M. R.; Bunner, A. E.; Harper J. F. An Isotope Labeling Strategy for Quantifying the Degree of Phosphorylation at Multiple Sites in Proteins. J. Am. Soc. Mass Spectrom. 2004, 15, 647-653.

15. Previs, M. J.; VanBuren, P.; Begin, K. J.; Vigoreaux, J. O.; LeWinter, M. M.; Matthews, D. E. Quantification of Protein Phosphorylation by Liquid Chromatography and Mass Spectrometry. Anal. Chem. 2008, 80, 5864-5872.

16. Gruhler, A.; Olsen, J. V.; Mohammed, S.; Mortensen, P.; Faergeman, N. J.; Mann, M.; Jensen, O. N. Quantitative Phosphoproteomics Applied to the Yeast Pheromone Signaling Pathway. Mol. Cell. Proteom. 2005, 4 $310-327$.

17. Olsen, J. V.; Blagoev, B.; Gnad, F.; Macek, B.; Kumar, C.; Mortensen, P.; Mann, M. Global, In Vivo, and Site-Specific Phosphorylation Dynamics in Signaling Networks. Cell 2006, 127, 635-648.

18. Steen, H.; Jebanathirajah, J. A.; Springer, M.; Kirschner, M. W. Stable Isotope-Free Relative and Absolute Quantitation of Protein Phosphorylation Stoichiometry by MS. Proc. Natl. Acad. Sci. U.S.A. 2005, 102, 3948-3953.

19. Beynon, R. J.; Doherty, M. K.; Pratt, J. M.; Gaskell, S. J. Multiplexed Absolute Quantification in Proteomics Using Artificial QCAT Proteins of Concatenated Signature Peptides. Nat. Methods 2005, 2, 587-589.

20. Pratt, J. M.; Simpson, D. M.; Doherty, M. K.; Rivers, J.; Gaskell, S. J.; Beynon, R. J. Multiplexed Absolute Quantification for Proteomics Using Concatenated Signature Peptides Encoded by QconCAT Genes. Nat. Protocols 2006, 1, 1029-1043.

21. Chu, M. L. H.; Chavas, L. M. G.; Douglas, K. T.; Eyers, P. A.; Tabernero, L. Crystal Structure of the Catalytic Domain of the Mitotic Checkpoint Kinase Mps1 in Complex with SP600125. J. Biol. Chem. 2008, 283, 21495-21500.

22. Eyers, C. E.; Simpson, D. M.; Wong, S. C. C.; Beynon, R. J.; Gaskell, S. J. QCAL: A Novel Standard for Assessing Instrument Conditions for Proteome Analysis. J. Am. Soc. Mass Spectrom. 2008, 19, 1275-1280.

23. Singh, S.; Springer, M.; Steen, J.; Kirschner, M. W.; Steen, H. FLEXIQuant: A Novel Tool for the Absolute Quantification of Proteins, and the Simultaneous Identification and Quantification of Potentially Modified Peptides. J. Proteome Res. 2009, 8, 2201-2210. 